\title{
Identification and expression profiles of sRNAs and their biogenesis and action-related genes in male and female cones of Pinus tabuliformis
}

\author{
Shi-Hui Niu, Chang Liu, Hu-Wei Yuan, Pei Li, Yue Li and Wei Li
}

\begin{abstract}
Background: Small RNA (sRNA) play pivotal roles in reproductive development, and their biogenesis and action mechanisms are well characterised in angiosperm plants; however, corresponding studies in conifers are very limited. To improve our understanding of the roles of sRNA pathways in the reproductive development of conifers, the genes associated with sRNA biogenesis and action pathways were identified and analysed, and sRNA sequencing and parallel analysis of RNA ends (PARE) were performed in male and female cones of the Chinese pine (Pinus tabuliformis).

Results: Based on high-quality reference transcriptomic sequences, 21 high-confidence homologues involved in sRNA biogenesis and action in P. tabuliformis were identified, including two different DCL3 genes and one AGO4 gene. More than $75 \%$ of genes involved in sRNA biogenesis and action have higher expression levels in female than in male cones. Twenty-six microRNA (miRNA) families and 74 targets, including 46 24-nt sRNAs with a 5' A, which are specifically expressed in male cones or female cones and probably bind to AGO4, were identified.

Conclusions: The sRNA pathways have higher activity in female than in male cones, and the miRNA pathways are the main sRNA pathways in P. tabuliformis. The low level of 24-nt short-interfering RNAs in conifers is not caused by the absence of biogenesis-related genes or AGO-binding proteins, but most likely caused by the low accumulation of these key components. The identification of sRNAs and their targets, as well as genes associated with sRNA biogenesis and action, will provide a good starting point for investigations into the roles of sRNA pathways in cone development in conifers.
\end{abstract}

Keywords: Pinus tabuliformis Carr, miRNA, siRNA, Parallel analysis of RNA ends (PARE), Male cones, Female cones

\section{Background}

The functional differentiation and adaptability to different environments of cells and tissues harbouring the same genetic material are dependent on epigenetic regulation at different levels. Small RNA (sRNA)-mediated gene silencing and chromatin modification play important roles in regulation [1]. The sRNA pathways in plants mainly include the microRNA (miRNA) and shortinterfering RNA (siRNA) pathways [2]. According to the biogenesis and action mechanisms of sRNAs, the siRNA

\footnotetext{
* Correspondence: bjfuliwei@bjfu.edu.cn
National Engineering Laboratory for Forest Tree Breeding, College of
Biological Science and Technology, Beijing Forestry University, Beijing

* Correspondence: bjfuliwei@bjfu.edu.cn
National Engineering Laboratory for Forest Tree Breeding, College of
Biological Science and Technology, Beijing Forestry University, Beijing

* Correspondence: bjfuliwei@bjfu.edu.cn
National Engineering Laboratory for Forest Tree Breeding, College of
Biological Science and Technology, Beijing Forestry University, Beijing, 100083 People's Republic of China
}

(C) 2015 Niu et al. Open Access This article is distributed under the terms of the Creative Commons Attribution 4.0 International License (http://creativecommons.org/licenses/by/4.0/), which permits unrestricted use, distribution, and reproduction in any medium, provided you give appropriate credit to the original author(s) and the source, provide a link to the Creative Commons license, and indicate if changes were made. The Creative Commons Public Domain Dedication waiver (http://creativecommons.org/publicdomain/zero/1.0/) applies to the data made available in this article, unless otherwise stated. natural-antisense siRNA (natsiRNA) and RNA-directed DNA methylation (RdDM) pathways [3].

The miRNAs are a family of small endogenous noncoding single-stranded RNA molecules that regulate gene expression posttranscriptionally by directing mRNA degradation or translational repression and control many biological functions, including development and tissuespecific processes in both plants and animals $[4,5]$. Plant miRNAs are generally 21 nucleotides long and regulate endogenous gene expression by recruiting silencing factors assembled into the RNA-induced silencing complex (RISC) to complementary binding sites in target transcripts [6, 7]. In most studied plants, such as Arabidopsis 
[8], rice [9], tomato [10], soybean [11], peanut [12], apple [13], miRNAs are the second most abundant sRNAs, followed by siRNAs [14]. siRNAs are distinguished from miRNAs in that they are derived from double-stranded RNA precursors. In plants, 24-nt siRNAs are associated with DNA methylation through the $\mathrm{RdDM}$ pathway at homologous loci guided by AGO4 proteins [15-18].

The sRNAs play a pivotal role in flower transformation and development $[19,20]$. miR156 participates in ambient temperature-responsive flowering [21] and male fertility [22], miR159 controls anther development [23, 24] and pollen tube-synergid interaction [25], miR172 mediates sex determination and floral meristem determinacy [26-28], miR319 is required for petal development [29], and miR396 is involved in pistil development $[30,31]$. Although there has been much work on the reproductive regulatory roles of miRNAs, there has been less emphasis on siRNAs. However, there is evidence that 24-nt siRNAs are probably critical in the regulation of flowering time [32], anthers [33], petals [34] and embryonic [35] development.

Despite this broad knowledge of sRNA biogenesis and the action mechanisms underlying growth and development of angiosperm plants, there is still a considerable lack of corresponding research on gymnosperms. With the popularisation of next-generation sequencing technology, sRNA sequencing and identification were also performed for some conifers $[9,36]$. The sRNA expression profiles of infectious diseases [37], somatic embryonic induction and germination $[38,39]$, and male and female gametophytes $[40,41]$ were analysed in different conifer trees. However, these studies focused mainly on changes in expression of specific sRNAs, while research on the sRNA biogenesis and action pathways is very limited.

To improve our understanding of the roles of sRNA pathways in male and female cones of Pinus tabuliformis, the genes associated with sRNA biogenesis and action pathways were identified and analysed, and highthroughput sequencing of sRNAs and degradome tags of $P$. tabuliformis male and female cones was performed. These data provide compelling new insights into the regulation of sRNA pathways involved in male and female cone development in P. tabuliformis.

\section{Results}

Identification of homologues involved in SRNA biogenesis and action in P. tabuliformis

The sRNA biogenesis and action pathways are well defined in Arabidopsis [3]. Through a Blast search of the $P$. tabuliformis transcriptomic sequences [42] using the amino acid sequences of proteins from Arabidopsis, several highly similar sequences were selected and mapped to the Picea abies genome [43]. Specific screening primers were designed based on the longest sequence in each cluster to isolate the full-length sequences from the P. tabuliformis SMART cDNA library (Clonetech, USA). Finally, 24 candidate genes with complete coding regions were isolated, and the phylogenetic relationships between these $P$. tabuliformis genes and those of other land plants were inferred using the ML method. Surprisingly, the sRNA pathway genes were highly conserved during evolution, except for methyltransferases involved in the anRdDM pathway (Additional file 1). Twenty-one high-confidence homologues involved in sRNA biogenesis and action in $P$. tabuliformis were identified (Table 1).

\section{Two different $D C L 3$ genes exist in conifers}

DCL enzymes are large proteins that catalyse primary sRNA transcript cleavage and produce mature sRNAs of different sizes [44]. Four different AtDCL enzymes were found in Arabidopsis and were divided into four groups, corresponding to DCLs from other plants. All four classes of DCLs exist in P. tabuliformis, indicating that they evolved before the divergence of angiosperms and gymnosperms (Additional file 1).

Different DCLs specifically process precursor transcripts into differently sized sRNAs. DCL1 and DCL4 generate 21-nt sRNAs, DCL2 generates 22-nt sRNAs, while DCL3 generates 24-nt sRNAs [45]. In angiosperms, the 24-nt sRNAs are the major endogenous sRNAs [9]; however, their levels are substantially lower in gymnosperms [43]. DCL3 was once considered to be absent in gymnosperm plants [46], but later studies suggest multiple DCL3 members exist in conifers [47].

Our results demonstrated two different $D C L 3$ genes in P. tabuliformis (Table 1, Fig. 1). The identities between the PtDCL3a and PtDCL3b cDNA sequences are only $68.5 \%$; however, the identity of PtDCL3a to its Pinus taeda and Picea abies homologues are $98 \%$ and $94 \%$, respectively, while the identity of PtDCL3b to its homologues are $97.0 \%$ and $93 \%$, respectively. These results indicate that $D C L 3 a$ and $D C L 3 b$ were separated for a long time before the divergence of conifer species.

\section{The AGO4s binding to the 24-nt DCL3-derived siRNAs were conserved during land plant evolution}

AGO proteins are key components of the RNA-induced silencing complex (RISC) [48, 49]. Phytogenetic analyses showed that plant AGO proteins group into three clades (Fig. 2a). Five AGOs were found in P. tabuliformis. PtAGO1, 5, and 10 belong to the AGO1 clade, and PtAGO4 and PtAGO7 belong to the AGO4 and AGO7 clades, respectively (Fig. 2b). The catalytic DDH amino acid core in the PIWI domain of land plant AGOs was extremely conserved (Fig. 2c).

Despite the fact that 24-nt DCL3-derived siRNAs are only present at very low levels in conifers [43] and that 
Table 1 The sRNA pathway genes in Pinus tabuliformis

\begin{tabular}{|c|c|c|c|c|c|}
\hline At gene & Locus & Pt homolog & NCBI NO. & Protein & Function \\
\hline AtHST & At3g05040 & PtHST & KJ711062 & 1195 & Exprotin-5 homolog \\
\hline AtHEN1 & At4g29160 & PtHEN1 & KJ711060 & 977 & sRNA-sprecific methyltransferase \\
\hline AtDRB4 & At3g62800 & PtDRB4 & KJ711042 & 550 & nuclear dsRNA-binding protein \\
\hline AtHYL1 & At1g09700 & PtHYL1 & KJ711063 & 485 & nuclear dsRNA-binding protein \\
\hline AtSGS3 & At5g23570 & PtSGS3 & KJ711106 & 776 & Coiled-coil protein \\
\hline AtRDR1 & At1g14790 & PtRDR1 & KJ711100 & 1726 & RNA-dependent RNA polymerase \\
\hline AtRDR2 & At4g11130 & PtRDR2 & KJ711101 & 1189 & RNA-dependent RNA polymerase \\
\hline AtRDR6 & At3g49500 & PtRDR6 & KJ711102 & 1123 & RNA-dependent RNA polymerase \\
\hline AtDCL1 & At1g01040 & PtDCL1 & KJ711036 & 2126 & Rnase III \\
\hline AtDCL2 & At3g03300 & PtDCL2 & KJ711037 & 1435 & Rnase III \\
\hline AtDCL3 & At3g43920 & PtDCL3a & KJ711038 & 1871 & Rnase III \\
\hline AtDCL3 & At3g43920 & PtDCL3b & KJ711039 & 1792 & Rnase III \\
\hline AtDCL4 & At5g20320 & PtDCL4 & KJ711040 & 1716 & Rnase III \\
\hline AtNRPDIa & At1g63020 & PtNRPD1a & KJ711089 & 1856 & DNA-dependent RNA polymerase \\
\hline AtNRPD1b & At2g40030 & PtNRPD1b & KJ711090 & 2530 & DNA-dependent RNA polymerase \\
\hline AtNRPD2 & At3g23780 & PtNRPD2 & KJ711091 & 1348 & DNA-dependent RNA polymerase \\
\hline AtAGO1 & At1g48410 & PtAGO1 & KJ710984 & 1144 & RNA slice \\
\hline AtAGO2 & At1g31280 & NA & & & RNA slice \\
\hline AtAGO3 & At1g31290 & NA & & & RNA slice \\
\hline AtAGO4 & At2g27040 & PtAGO4 & KJ710986 & 930 & RNA slice \\
\hline AtAGO5 & At2g27880 & PtAGO5 & KJ710987 & 1097 & RNA slice \\
\hline AtAGO6 & At2g32940 & PtAGO4 & KJ710986 & 930 & RNA slice \\
\hline AtAGO7 & At1g69440 & PtAGO7 & KJ710988 & 1127 & RNA slice \\
\hline AtAGO9 & At5g21150 & PtAGO4 & KJ710986 & 930 & RNA slice \\
\hline AtAGO10 & At5g43810 & PtAGO10 & KJ710985 & 955 & RNA slice \\
\hline AtCMT3 & At1g69770 & NA & & & Methyltransferase \\
\hline AtDRM2 & At5g15380 & NA & & & Methyltransferase \\
\hline AtMET1 & A5t49160g & NA & & & Methyltransferase \\
\hline
\end{tabular}

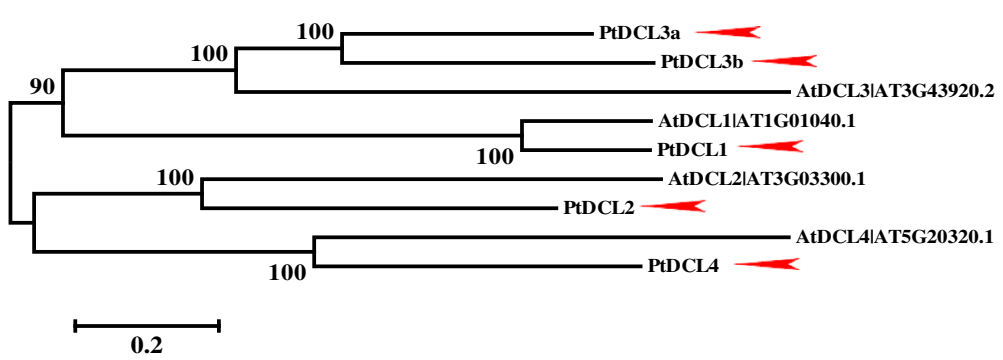

Fig. 1 Phylogenetic analysis of PtDCL and AtDCL proteins. The figures show an unrooted maximum likelihood tree based on amino acid sequences. The gene names and IDs are provided to the right of each branch. The horizontal branch lengths are proportional to the estimated number of amino acid substitutions per residue. Bootstrap values were obtained from 1000 bootstrap replicates. The arrows indicate $P$. tabuliformis genes investigated in this study. The ML tree of DCL proteins from 42 land plants is shown in Additional file 1 
$\mathbf{a}$

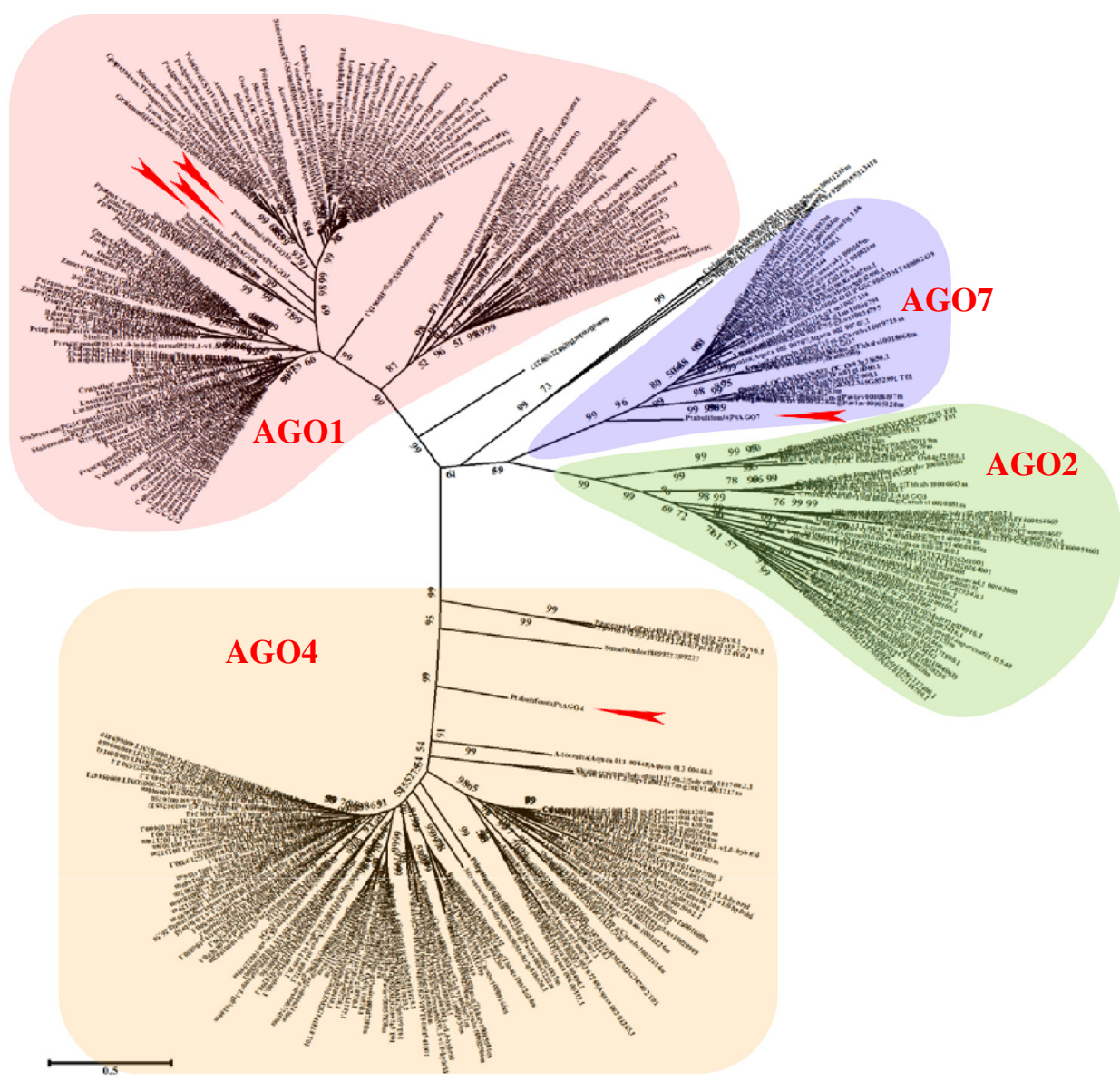

b

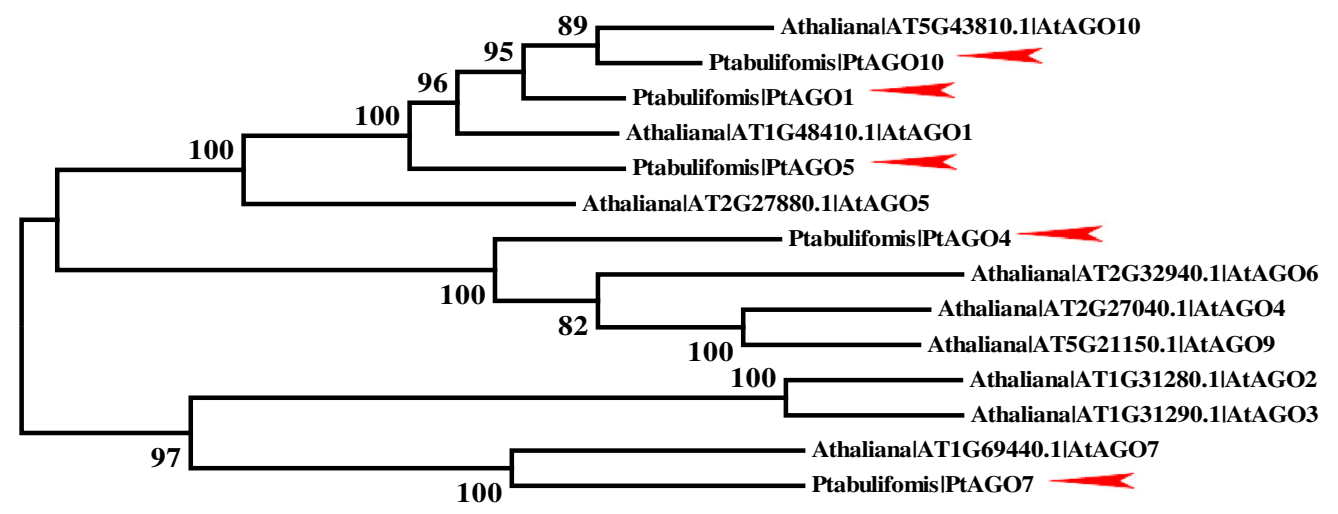

0.2

c

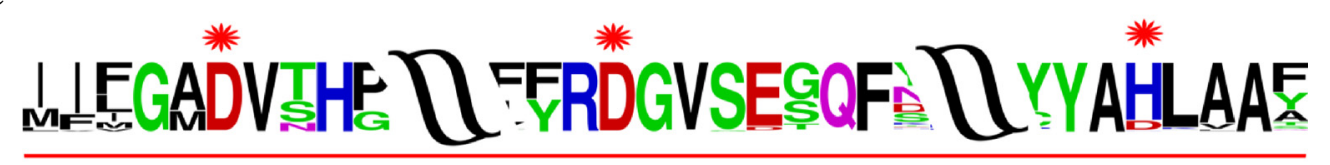

PIWI domain

Fig. 2 (See legend on next page.) 
(See figure on previous page.)

Fig. 2 Phylogenetic analysis of AGO proteins in land plants. (a), The figures show an unrooted ML tree based on the amino acid sequences of all AGO proteins in land plants. (b), The figures show an unrooted ML tree based on the PtAGOs and AtAGOs. The horizontal branch lengths are proportional to the estimated number of amino acid substitutions per residue. Bootstrap values were obtained from 1000 bootstrap replicates. The arrows indicate the $P$. tabuliformis genes investigated in this study. (c) The catalytic DDH amino acid core in the PIWI domain of land plant AGOs. The sizes of letters represent the residue frequency of each site

the AGO4 clade ago mutants in Arabidopsis (ago4, ago6, ago9) have no obvious developmental defects [48], AGO4s were conserved during land plant evolution. Moreover, the number, position, and size of exons of AGO4 homologues in land plants remained surprisingly consistent (Fig. 3). Greater efforts are needed to understand the specific role of AGO4 in species maintenance and evolution.

The sRNA biogenesis and action pathways have higher activity in female than in male cones of $P$. tabuliformis The expression profiles of genes involved in the sRNA biogenesis and action pathways in male and female cones were analysed. The results show that more than $75 \%$ of genes have higher expression levels in female than in male cones (Fig. 4a). These differences were confirmed by microarray data (Additional file 2). Interestingly, the female structures (carpels) in Arabidopsis also had similarly higher activities than those of the male structures (stamens) (Fig. 4b). Moreover, AGO1 had the highest expression level, and AGO4 and AGO10 were highly differentially expressed between male and female structures in both P. tabuliformis and Arabidopsis, indicating that a similar sRNA regulatory mechanism probably underlies the development of male and female structures in both gymnosperms and angiosperms.

sRNAs in male and female cones were then analysed by high-throughput sequencing. The results showed that 21-nt sRNAs were the major sRNAs in both male and female cones in P. tabuliformis, with more in female than male cones (Fig. 5). Proportionally, the male cones had relatively high levels of 24-nt sRNAs (Fig. 5), but AGO4, which plays a key role in the action of 24-nt sRNAs, was expressed at a very low level in male cones (Fig. 4a), indicating that both miRNA and siRNA pathways have higher activities in female than male cones.

\section{Identification of miRNAs and targets in male and female cones of $P$. tabuliformis}

To globally and directly identify miRNAs and miRNAdirected targets of cleavage, a parallel analysis of RNA ends (PARE), also known as degradome analysis, was applied. Twenty-six miRNA families and 74 targets were identified by sRNA sequencing and PARE analysis. Three novel miRNAs with unknown functions were isolated (Table 2, Additional file 3). When a two-fold change (FC) in expression was used to filter the differentially expressed miRNAs between male and female cones, 50 miRNAs were identified (Additional file 4). Eighteen genes had higher expression levels in male cones, while the other 32 miRNAs had higher expression levels in female cones (Additional file 4). This result is consistent with the sRNA biogenesis and action pathways having higher activities in female than in male cones in P. tabuliformis (Fig. 4).

The completed coding DNA sequences (CDSs) of 36 target genes were isolated, of which 20 miRNA targets were conserved in the evolution of conifers and angiosperms (Table 2). The important miRNA targets involved in angiosperm reproductive development, such as miR156/miR529-SPLs [50], miR159-

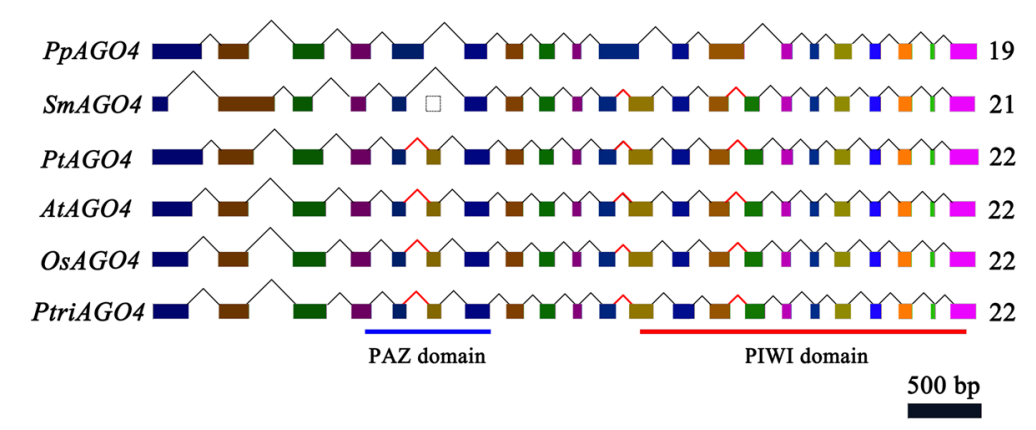

Fig. 3 The gene structures and functional domains of land plant AGO4 genes. The AGO4 homologous structures in P. patens, S. moellendorffii, P. tauliformis, A. thaliana, $O$. sativa and $P$. trichocarpa. Coloured boxes represent different exons. The total numbers of exons are shown to the right side of the figure 


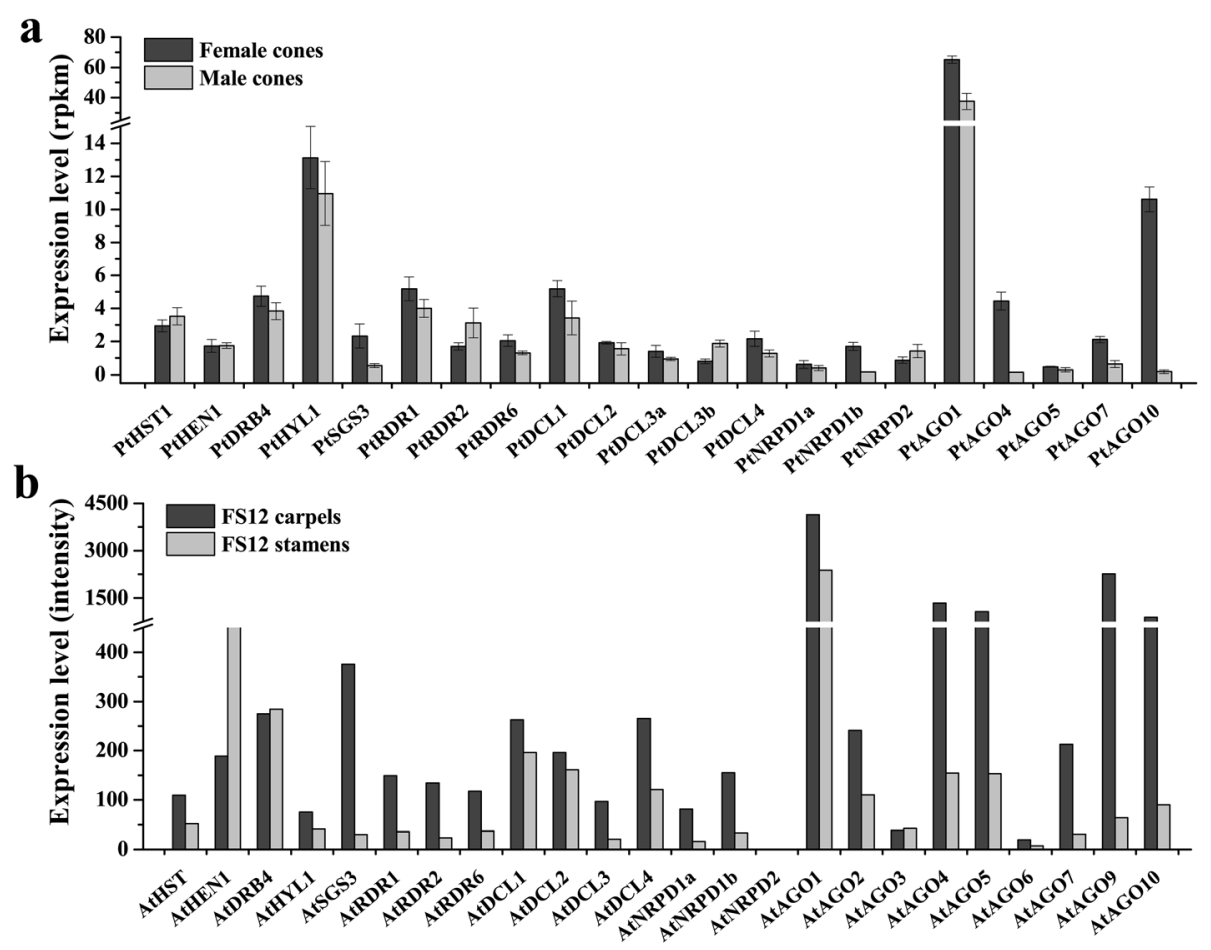

Fig. 4 The expression patterns of genes involved in sRNA biogenesis and action pathways in male and female reproductive structures of $P$. tabuliformis and A. thaliana. (a), The expression patterns of $P$. tabuliformis genes in male and female cones. Bars indicate the means and standard errors of three biological replicates. (b), The expression patterns of $A$. thaliana genes in the stamens and carpels. FS12 indicates flower stage 12. The expression data were downloaded from the A. thaliana database (http://jsp.weigelworld.org/expviz/expviz.jsp)

MYBs [51], miR172-AP2Ls [52], miR319-TCPs [53] and miR396-GRFs [31], also exist in P. tabuliformis (Table 2, Additional file 5). The miR396-GRFs were previously found to be required for coordination of cell division and differentiation during leaf development [54, 55], and recent studies have shown that they also play a role in reproductive development $[30,31]$. We isolated three GRF homologues from $P$. tabuliformis, namely PtGRF1-3, and miR396 mediated cleavage of the PtGRFs and regulated PtGRF mRNA accumulation (Fig. 6).
Identification of 24-nt sRNAs containing a 5' " $\mathrm{A}$ " terminal differentially expressed between male and female cones in $P$. tabuliformis

Compared with the miRNA pathway, the role of the 24nt siRNA-mediated RdDM pathway in the reproductive development of plants is largely unknown [48]. Only one AGO4 homologue, the key component of RISC associated with 24-nt siRNAs, was found in P. tabuliformis (Table 1, Fig. 2). Because AGO4 was revealed to predominantly bind 24-nt sRNAs with a 5' A [56], the 24-nt sRNAs containing 5' "A" termini differentially expressed
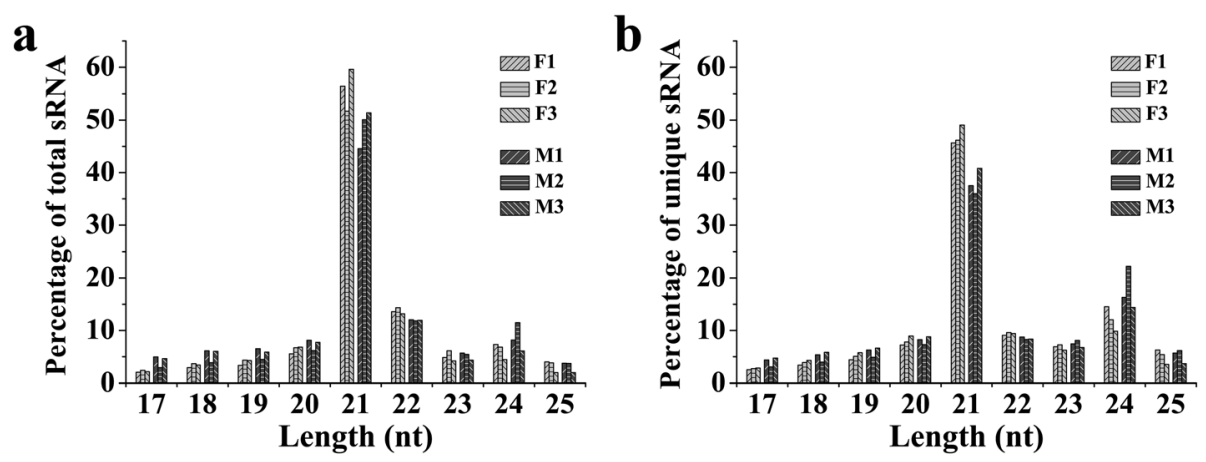

Fig. 5 The sRNA length distribution in male and female cones of $P$. tabuliformis. (a) The length distribution of total sRNAs. (b) The length distribution of unique sRNAs. M1-3 and F1-3 indicate the three libraries of male cones and female cones, respectively 
Table 2 Experimental identified miRNA targets in P. tabuliformis

\begin{tabular}{|c|c|c|c|c|}
\hline miRNA family & Target & Protein & Conserved target / miRNA sequence & Action sites \\
\hline miR156 & comp75271_c0_seq2 & PtSPL1 & {$[50]$} & Flowering \\
\hline $\operatorname{miR} 156$ & Iw_isotig09062 & PtSPL3 & {$[50]$} & Flowering \\
\hline miR529 & comp85892_c0_seq1 & PtSPL2 & {$[76]$} & Flowering \\
\hline miR159 & Iw_hbkxs4402jlyd6 & PtMYB33 & {$[51]$} & Flowering \\
\hline miR162 & comp74382_c0_seq3 & PtDCL1 & {$[52]$} & sRNA \\
\hline miR172 & comp64707_c0_seq1 & PtAP2L3 & {$[52]$} & Flowering \\
\hline miR172 & Iw_isotig05156 & PtAP2L2 & {$[52]$} & Flowering \\
\hline miR172 & Iw_isotig06154 & PtAP2L1 & {$[52]$} & Flowering \\
\hline miR319 & Iw_isotig09509 & PtTCP2 & {$[53]$} & Flowering \\
\hline miR319 & Iw_isotig09013 & PtERF1 & 5'-TTGGACTGAAGGGAGCTCC-3' & \\
\hline miR166 & comp65619_c0_seq2 & $\mathrm{PtHB}^{*}$ & {$[77]$} & Vascular \\
\hline miR166 & comp78056_c0_seq1 & PtHB3* & {$[77]$} & Vascular \\
\hline $\operatorname{miR} 166$ & comp83755_c0_seq1 & PtHB4 & {$[77]$} & Vascular \\
\hline miR166 & Iw_isotig05204 & PtHB2 & {$[77]$} & Vascular \\
\hline miR169 & comp77240_c0_seq3 & PtNF-YA7 & {$[78]$} & Root \\
\hline miR171 & comp65826_c0_seq1 & PtHAM1 & {$[79]$} & Meristem \\
\hline miR171 & Iw_hbkxs4402gb5ou & PtHAM2 & {$[79]$} & Meristem \\
\hline miR391 & comp48694_c0_seq1 & unknown & 5'-TACGCAGGAGAGATGACACCG-3' & \\
\hline miR391 & Iw_isotig02711 & unknown & 5'-TACGCAGGAGAGATGACACCG-3' & \\
\hline miR394 & Iw_isotig14380 & PtKRF2 & {$[80]$} & Stem cell \\
\hline miR396 & comp57471_c0_seq2 & PtGRF2* & {$[31]$} & Flower/Pistil \\
\hline miR396 & comp73392_c0_seq1 & PtGRF1 & {$[31]$} & Flower/Pistil \\
\hline miR396 & Iw_isotig04039 & PtGRF3 & {$[31]$} & Flower/Pistil \\
\hline miR408 & comp20033_c0_seq1 & PtSINAT1 & 5'-TGCACTGCCTCTTCCCTGGCT-3' & \\
\hline miR408 & Iw_isotig03980 & PtAPRN & 5'-TGCACTGCCTCTTCCCTGGCT-3' & \\
\hline miR482 & comp10992_c0_seq1 & PtNBS1 & {$[81]$} & Defense \\
\hline miR482 & comp2059_c0_seq1 & unknown & 5'-ТСТTТCСТАСТССТССCA-3' & \\
\hline miR482 & comp270247_c0_seq1 & unknown & 5'-ТCTTСССТАСТССТСССАТTCC-3' & \\
\hline miR482 & comp43645_c0_seq1 & unknown & 5'-TTTCCTACTCCTCCCAAGCCCA-3' & \\
\hline miR482 & comp57920_c0_seq1 & unknown & 5'-TTCCTACTCCTCCCAAGCCCA-3' & \\
\hline miR482 & comp59077_c0_seq2 & unknown & 5'-TCTTGCCTACCCCTCCCATTCC-3' & \\
\hline miR482 & comp66603_c0_seq1 & unknown & 5'-TTCCTACTCCTCCCAAGCCCA-3' & \\
\hline miR482 & comp76079_c0_seq1 & unknown & 5'-ТCTТСССТАСТССТСССАТTCC-3' & \\
\hline miR482 & comp80951_c0_seq5 & unknown & 5'-TTTCCTACTCCTCCCAAGCCCA-3' & \\
\hline miR482 & Iw_isotig06642 & PtKRF3 & 5'-ТСТТСССТАСТССТСССАТTCC-3' & \\
\hline miR482 & Iw_isotig09777 & unknown & 5'-ТСТTСССТАСТССТCCCATTCC-3' & \\
\hline miR482 & Iw_isotig12233 & unknown & 5'-ТТСССТАТТССТСССАТТССТА-3' & \\
\hline miR482 & Iw_isotig17369 & unknown & 5'-ТCTTCCСТAСТССТCCCATTCC-3' & \\
\hline miR482 & Iw_isotig25482 & unknown & 5'-TTTCCTACTCCTCCCAAGCCCA-3' & \\
\hline miR946 & comp74586_c0_seq1 & un known & 5'-CAGCCCTTCTCCTATCCACAAC-3' & \\
\hline miR947 & comp58863_c0_seq2 & unknown & 5'-CATCGGAATCTGTTACTGTTTC-3' & \\
\hline miR947 & comp69066_c0_seq2 & unknown & 5'-CATCGGAATCTGTTACTGTTTC-3' & \\
\hline $\operatorname{miR947}$ & Iw_hbkxxs4402jaz6z & unknown & 5'-CATCGGAATCTGTTACTGTTTC-3' & \\
\hline miR947 & Iw_isotig08583 & unknown & 5'-CATCGGAATCTGTTACTGTTTC-3' & \\
\hline
\end{tabular}


Table 2 Experimental identified miRNA targets in P. tabuliformis (Continued)

\begin{tabular}{|c|c|c|c|}
\hline miR949 & comp29204_c0_seq1 & unknown & 5'-TCTCCGGGAATCCAATGCGCCT-3' \\
\hline miR949 & comp4036_c0_seq1 & unknown & 5'-TCTCCGGGAATCCAATGCGCCT-3' \\
\hline miR950 & comp314883_c0_seq1 & NB-ARC & 5'-TAACATCTGGGCCACGAGGGTT-3' \\
\hline miR950 & Iw_hbkxs4402g5r7f & unknown & 5'-TCACATCTGGGCCACGATGGTT-3' \\
\hline miR951 & comp77599_c0_seq2 & unknown & 5'-TGTTCTTGACGTCTGGACCACG-3' \\
\hline miR951 & comp79416_c0_seq1 & unknown & 5'-TGTTCTTGACGTCTGGACCACG-3' \\
\hline miR951 & comp79471_c2_seq6 & unknown & 5'-TCGGCCTCAAATGTTAGGAGAA-3' \\
\hline miR951 & Iw_hbkxs4401es9b| & unknown & 5'-TGTTCTTGACGTCTGGACCACG-3' \\
\hline miR1311 & Iw_isotig09685 & unknown & 5'-TCAGAGTITTGCCAGTTCCGCC-3' \\
\hline $\operatorname{miR} 1312$ & comp141994_c0_seq1 & PtGRF2* & 5'-TTTGGAGAGAAAATGGCCACT-3' \\
\hline $\operatorname{miR} 1312$ & comp78456_c0_seq1 & PtHB1 & 5'-TTTGGAGAGAAAATGGCCACT-3' \\
\hline miR1313 & comp70891_c0_seq2 & PtLRK1 & 5'-TACCACTGAAATTATTGTTCG-3' \\
\hline $\operatorname{miR} 1314$ & comp14858_c0_seq1 & unknown & 5'-CCGGCCTCAAATGTTAGGAGAA-3' \\
\hline miR1314 & comp47488_c0_seq1 & unknown & 5'-CCGGCCTCAAATGTTAGGAGAA-3' \\
\hline miR1314 & comp62379_c0_seq1 & unknown & 5'-CCGGCCTCAAATGTTAGGAGAA-3' \\
\hline miR1314 & comp66316_c0_seq1 & unknown & 5'-CCGGCCTCAAATGTTAGGAGAA-3' \\
\hline $\operatorname{miR} 1314$ & comp67690_c0_seq1 & unknown & 5'-CCGGCCTCGAATGTTAGGAGA-3' \\
\hline miR1314 & comp77805_c0_seq6 & unknown & 5'-CCGGCCTCAAATGTTAGGAGAA-3' \\
\hline $\operatorname{miR} 1314$ & comp78314_c0_seq1 & PtRNAase & 5'-CCGGCCTCAAATGTTAGGAGAA-3' \\
\hline $\operatorname{miR} 1316$ & Iw_isotig01063 & PtLIP1* & 5'-TTCCATGCACAAACCATTGGA-3' \\
\hline miR1316 & Iw_isotig22693 & PtLIP1* & 5'-TTCCATGCACAAACCATTGGA-3' \\
\hline miR1316 & Iw_isotig25086 & PtLIP2 & 5'-TTCCATGCACAAACCATTGGA-3' \\
\hline miR1316 & Iw_isotig25889 & PtLIP1* & 5'-TTCCATGCACAAACCATTGGA-3' \\
\hline miR1448 & Iw_isotig17502 & unknown & 5'-TCTITCCAACGCCTCCCATACC-3' \\
\hline miR2111 & Iw_isotig01996 & PtKRF1 & 5'-TAATCTGCATCCTGAGGTTTG-3' \\
\hline miR2118 & comp35426_c0_seq1 & unknown & 5'-TTCCСТАТТССАСССАТСCCAT-3' \\
\hline miR3710 & comp76797_c0_seq3 & unknown & 5'-TGAACAATGCCCACCCTTCATC-3' \\
\hline new & comp333751_c0_seq1 & unknown & 5'-TGACATTGTAAAATACGGGAAT-3' \\
\hline new & comp54693_c0_seq1 & unknown & 5'-TCAGGGCCTCGGTGGTTAATG-3' \\
\hline new & comp69194_c0_seq1 & PtmTERF1 & 5'-TAATGCTTCACCCTCAATGCC-3' \\
\hline
\end{tabular}

The miRNAs that shown in the table were isolation and sequencing from at least two independent libraries and the targets cleavage by miRNAs were identified by PARE analysis. * indicate the unigenes with same name were found as same gene after cloned

between male and female cones of $P$. tabuliformis were identified. Eleven and 35 sRNAs specifically expressed in male and female cones, respectively, were isolated (Additional file 6). The functional identification of these 24-nt sRNAs in reproductive development will be instructive to our future research.

\section{Discussion}

The sRNA-mediated transcriptional regulation of genes, including the miRNA and siRNA pathways, is an important epigenetic regulatory mechanism in plants [1]. In this study, we first isolated the key regulatory factors involved in miRNA and siRNA biogenesis and action in $P$. tabuliformis. Phylogenetic analysis indicated that sRNA pathways were very ancient regulatory mechanisms during the evolution of land plants, and most homologous genes, such as DCLs, AGOs and RDRs, had already diverged in the primitive vascular plants. However, the siRNA pathways probably evolved later than the miRNA pathways. The sRNA binding and guiding protein AGOs and the 24nt siRNA-mediated DNA methylation catalytic genes have expanded and diversified in angiosperms [57].

In addition to the sRNA target genes, the sRNA biogenesis and action pathways also play important roles in the regulation of growth and development in plants [58, 59]. The expression profiles of the sRNA biogenesis and action pathway genes and sRNA sequencing indicated that the miRNA pathway is the main sRNA pathway in male and female cones of P. tabuliformis. Previous studies showed that the siRNA pathway has weak activity in other organs 


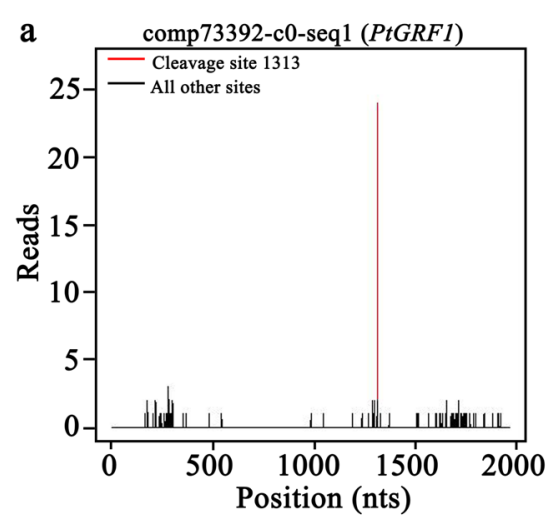

c

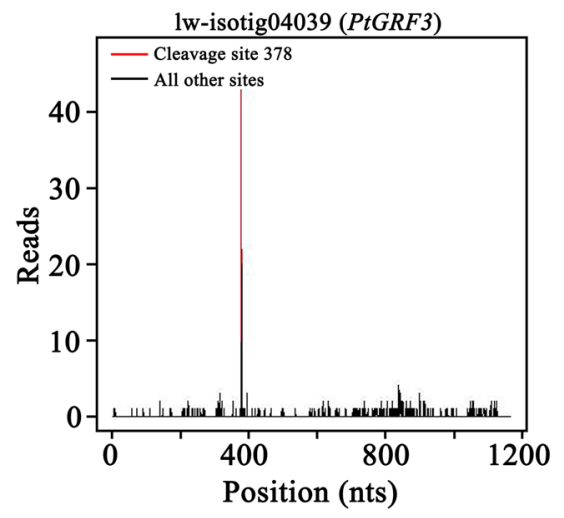

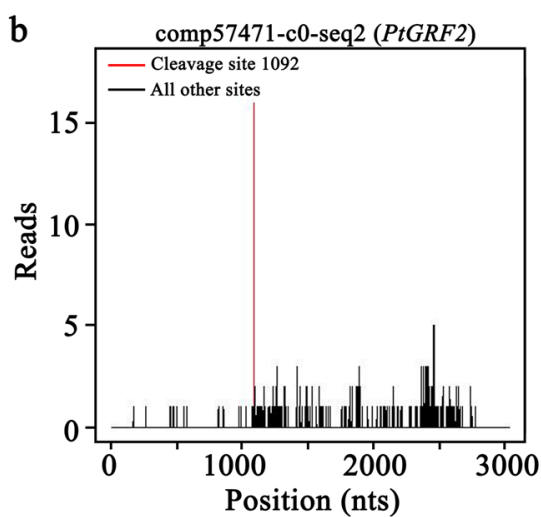

d

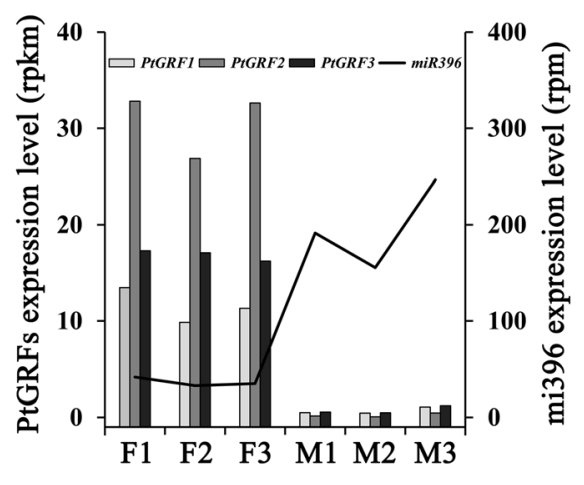

Fig. 6 The cleavage and expression patterns of miR396 targets in male and female cones of $P$. tabuliformis. (a)-(c) Experimental identification of cleaved miR396 targets by miR396. (d) The expression patterns of miR396 and its targets in male and female cones of P. tabuliformis

compared with cones $[35,43]$. In angiosperms, the miRNA pathway is also the most important sRNA pathway in reproductive regulation [20]. Based on sRNA sequencing and PARE analysis, the cleavage of 74 target sequences by 26 corresponding miRNA families was identified. The complete CDS of 36 genes from these target sequences were cloned, while other genes were difficult to obtain by PCR as the mRNA of these genes was almost completely degraded by the high abundance of related miRNAs (average RPM > 3700) in the cones of P. tabuliformis. The roles of turn off of these genes in reproductive development remain unclear. It is noteworthy that we found that at least a portion of these genes were probably non-coding RNAs, and may be indirectly involved in developmental regulation.

Our results showed that the important miRNAs and their targets involved in angiosperm reproductive development, such as miR156/miR529-SPLs [50], miR159MYBs [51], miR172-AP2Ls [52], miR319-TCPs [53] and miR396-GRFs [31], coevolved and have an ancient evolutionary history, similar to the sRNA pathways, such as miR156 and miRNA319, which have evolved in moss plants [60]. These miRNA-target-mediated regulatory pathways may have also coevolved as a "package", as MYB33 is the target of miR159, which is predominantly expressed in the male reproductive structures in different species [23, 24].

DNA methylation is involved in the control of all genetic functions including transcription, replication, DNA repair, gene transposition and cell differentiation in plants [61]. It is a common and very ancient epigenetic regulatory mechanism in plants that is found in the DNA of all archegoniates investigated; however, the degree and features of DNA methylation are species-, tissue-, organelle- and age-specific [61]. 24-nt siRNAmediated site-specific DNA methylation through the RdDM pathway is an important DNA methylation mechanism [62]. Previous studies suggested that gymnosperms have lower DNA methylation levels than those of flowering plants [63], which may be associated with the high degree of conservation and low morphological diversity between conifer species [43]. The 24-nt sRNAs involved in RdDM only represent a small proportion of all sRNAs in conifers [35, 43], but the proportions are opposite in the flowering plants [9]. Therefore, some researchers have speculated that the 
RdDM pathway in conifers is incomplete [46]. Our results have shown that, except for methyltransferase, all RdDM pathway components are present and conserved in $P$. tabuliformis, including PtDCL3, PtAGO4, PtRDR2, PtHEN1, PtNRPD1a, PtNRPD1b and PtNRPD2. The low level of 24-nt sRNAs is not because of a lack of biogenesis enzymes. The real reason may be, the low expression levels of RDR2-NRPD1a-DCL3 coding genes necessary for 24-nt sRNA accumulation.

AGO proteins are sRNA binding and guiding proteins and the most important proteins downstream of the sRNA pathways [64]. Despite the RdDM pathway having only weak activity in conifers, the components of RdDM were still conserved at a high degree through time. The structures of AGO4 in moss, lycophyte, gymnosperm and angiosperm plants maintain a high level of consistency. Interestingly, the role of RdDM in mosses and lycophytes is unclear, as the ago4 mutant has no obvious developmental defects $[65,66]$ and the evolutionary significance and selective pressure of the conservation of AGO4 and $\mathrm{RdDM}$ is difficult to understand. Some evidence indicates that the absence of $A G O 4$ makes the plants more sensitive to disease [65]. Investigating the role of PtAGO4 in $P$. tabuliformis in disease resistance may be valuable for understanding the role of RdDM in evolution and may facilitate disease resistant breeding of $P$. tabuliformis.

We found 46 24-nt sRNAs with a 5' A that probably bind to AGO4 [56]. They were specifically expressed in either male cones or female cones, and more than $75 \%$ of these sRNAs have significant accumulation in female cones but were not detected in all male samples. This is consistent with the higher activity of sRNA biogenesis and action pathway genes in female cones compared with male cones of $P$. tabuliformis. Because of the huge genome size, the analysis of large-scale genome methylation is difficult in conifers, and the function of these specifically expressed 24-nt sRNAs is unclear and deserves more attention in future studies.

\section{Conclusions}

Based on high-quality reference transcriptome sequences [42], 21 high-confidence homologues involved in sRNA biogenesis and action in P. tabuliformis were identified. Phylogenetic analysis indicated that the sRNA pathways are highly conserved from mosses and ferns to higher plants. The expression profiles of these genes suggested that the sRNA pathways have higher activities in female than in male reproductive structures. In contrast to the angiosperms [14], both biogenesis- and action-related gene expression and sRNA sequencing revealed that the miRNAs are the most abundant sRNAs in P. tabuliformis, rather than siRNAs. In this study, 26 miRNA families and the miRNA-directed cleavage of 74 corresponding targets were identified though correlation analysis of sRNA and
PARE sequencing data. The miRNAs and their targets participating in reproductive development in angiosperms, such as miR156-SPLs, miR159-MYBs, miR172- AP2Ls, miR319-TCP and miR396-GRFs, were also found in $P$. tabuliformis. They have ancient evolutionary histories similar to the sRNA pathways.

In conifers, the low level of 24-nt DCL3-derived siRNAs was not caused by the absence of DCL3 and AGO4. Two DCL3 genes and one AGO4 gene were found in P. tabuliformis, its ortholog PgAGO in Picea glauca [67] was previously identified. Forty six 24 nt sRNAs with a $5^{\prime}$ A, which probably bind to AGO4, specifically expressed in either male or female cones were isolated. The specific, highly expressed 24-nt sRNAs identified in conifers will provide a good starting point for investigations into the function and evolution of siRNAs in conifers.

\section{Methods}

\section{Plant material and sample collection}

P. tabuliformis immature male and female cones were collected from 3 individual trees selected at random (genetically distinct) in the botanic gardens in Beijing, China (116 $33.9116^{\prime} \mathrm{E}, 40^{\circ} 00.0861^{\prime} \mathrm{N}$ and $44 \mathrm{~m}$ a.s.l.). Cones were sampled at 11:00 am on April 21, 2013. Each experiment was performed with at least three biological replicates per event. Samples were immediately placed in liquid nitrogen in the field after collection and all samples were stored at $-80^{\circ} \mathrm{C}$ in the laboratory before analysis.

\section{Identification of homologues involved in SRNA pathways in P. tabuliformis}

Amino acid sequences of Arabidopsis thaliana genes (Table 1) were downloaded from the TAIR database (http://Arabidopsis.org). The protein sequences of Arabidopsis were used in queries to screen the P. tabuliformis transcriptome sequences (NCBI accession number SRA 056887) based on the TBLASTN method. The candidate sequences were selected and compared with other available conifer transcriptome sequences (http://dendrome.ucdavis.edu/resources/) and the Picea abies genome (http://congenie.org). The P. tabuliformis complete-length SMART cDNA library (Clonetech, USA) was screened using specific primers. The full-length sequences were obtained and compared with the original sequences. The nucleotide sequences of candidate genes were selected for preliminary phylogenetic analysis based on the NJ method using the MEGA software [68] and renamed.

\section{Phylogenetic analysis}

Homologues of 41 land plant species, which have been whole genome sequenced (http://phytozome.jgi.doe.gov), were selected for phylogenetic analysis. Multiple alignments of protein sequences were obtained using the MUSCLE software [69] and a maximum-likelihood tree, based on the 
JTT model, was generated using MEGA software [68]. Bootstrap values were obtained from 1000 replicates.

\section{sRNA sequencing and PARE analysis}

Total RNA isolation from samples and cDNA library construction were performed as described previously [39]. Pooled libraries were used for cluster generation on Illumina's Cluster Station (Illumina, San Diego, USA) and then sequenced on an Illumina Hiseq2000 at YQYK-BIO (Beijing, China) following the vendor's recommended protocol. The sRNA abundance was measured as reads per million reads (RPM). The PARE library construction and sequencing were performed as described previously [70, 71]. The identification of miRNA and miRNA-directed targets of cleavage though correlation analysis of sRNA and PARE sequencing results was performed as previously described [72, 73]. More details are available in the supplementary material (Additional file 7).

\section{Gene expression analysis}

RNA sequencing and gene expression analysis were described previously [74]. mRNA abundance was measured as reads per kilobase per million (RPKM) [75]. Each experiment was performed with at least three biological replicates per event. The mean RPKM of three biological replicates was compared among different samples.

\section{Identification of differentially expressed 24-nt sRNAs con-} taining a 5 ' "A" terminal between male and female cones The 24-nt sRNAs containing a 5' "A" terminal were extracted. Comparison of the expressions of these sRNAs was conducted between small RNA libraries of male and female cones. We first normalised the expression of sRNA in six libraries ( $\mathrm{F}$ and $\mathrm{M}$, three biological replicates each) to obtain the expression of reads per million reads (RPM). Then, the data were analysed using Fisher's exact test with a Bonferroni correction for multiple hypothesis testing. Those sRNAs with a $p$-value below 0.01 and specifically expressed in either male cones or female cones were isolated.

\section{Additional files}

Additional file 1: Phylogenetic relationships of land plant DCL-like, HEN1-like, HST-like, RDR-like and SGS3-like proteins. (DOCX 267 kb)

Additional file 2: The expression patterns of genes involved in sRNA biogenesis and action pathways in male and female reproductive structures of $P$. tabuliformis detected by microarray. (DOCX $105 \mathrm{~kb}$ )

Additional file 3: Experimental identification of cleaved miRNA targets. (DOCX $2271 \mathrm{~kb}$ )

Additional file 4: The differentially expressed miRNAs in male and female cones of $P$. tabuliformis. (DOCX $27 \mathrm{~kb}$ )
Additional file 5: Phylogenetic analysis of miR156/miR529, miR159, miR172, miR319 and miR396 regulated targets. (DOCX $98 \mathrm{~kb}$ )

Additional file 6: The differentially expressed 24-nt sRNAs containing a $5^{\prime}$ " $\mathrm{A}$ " terminal in male and female cones of $P$. tabuliformis. (DOCX $20 \mathrm{~kb}$ )

Additional file 7: Detailed description of methods. (DOCX $25 \mathrm{~kb}$ )

\section{Competing interests}

The authors declare that they have no competing interests.

\section{Authors' contributions}

SHN participated in the isolation of genes and drafted the manuscript. CL, HWY and PL participated in the samples preparation. SHN and YL participated in the design of the study and performed the statistical analysis. WL conceived of the study, and participated in its design and coordination and helped to draft the manuscript. All authors read and approved the final manuscript.

\section{Acknowledgements}

This work was supported by "The National Natural Science Foundation of China (NO. 31370657)" and "The Fundamental Research Funds for the Central Universities (NO. BLX2014-23)".

Received: 28 February 2015 Accepted: 27 August 2015

Published online: 15 September 2015

\section{References}

1. Henderson IR, Jacobsen SE. Epigenetic inheritance in plants. Nature. 2007:447(7143):418-24

2. Kallman T, Chen J, Gyllenstrand N, Lagercrantz U. A significant fraction of 21-nucleotide small RNA originates from phased degradation of resistance genes in several perennial species. Plant Physiol. 2013;162(2):741-54.

3. Eamens A, Wang MB, Smith NA, Waterhouse PM. RNA silencing in plants: yesterday, today, and tomorrow. Plant Physiol. 2008;147(2):456-68.

4. Pillai RS, Bhattacharyya SN, Artus CG, Zoller T, Cougot N, Basyuk E, et al. Inhibition of translational initiation by Let-7 MicroRNA in human cells. Science. 2005;309(5740):1573-6.

5. Bhattacharyya SN, Habermacher R, Martine U, Closs El, Filipowicz W. Relief of microRNA-mediated translational repression in human cells subjected to stress. Cell. 2006:125(6):1111-24.

6. Djuranovic S, Nahvi A, Green R. A parsimonious model for gene regulation by miRNAs. Science. 2011:331(6017):550-3.

7. Chen X. Small RNAs and their roles in plant development. Annu Rev Cell Dev Biol. 2009:25:21-44.

8. Rajagopalan R, Vaucheret $H$, Trejo J, Bartel DP. A diverse and evolutionarily fluid set of microRNAs in Arabidopsis thaliana. Genes Dev. 2006:20(24):3407-25

9. Morin RD, Aksay G, Dolgosheina E, Ebhardt HA, Magrini V, Mardis ER, et al. Comparative analysis of the small RNA transcriptomes of Pinus contorta and Oryza sativa. Genome Res. 2008;18(4):571-84

10. Moxon S, Jing R, Szittya G, Schwach F, Rusholme PR, Moulton V, et al. Deep sequencing of tomato short RNAs identifies microRNAs targeting genes involved in fruit ripening. Genome Res. 2008:18(10):1602-9.

11. Song QX, Liu YF, Hu XY, Zhang WK, Ma B, Chen SY, et al. Identification of miRNAs and their target genes in developing soybean seeds by deep sequencing. BMC Plant Biol. 2011;11(1):5.

12. Zhao CZ, Xia H, Frazier TP, Yao YY, Bi YP, Li AQ, et al. Deep sequencing identifies novel and conserved microRNAs in peanuts (Arachis hypogaea L.). BMC Plant Biol. 2010;10:3

13. Xia R, Zhu H, An YQ, Beers EP, Liu Z. Apple miRNAs and tasiRNAs with novel regulatory networks. Genome Biol. 2012;13(6):R47.

14. Lu C, Tej SS, Luo S, Haudenschild CD, Meyers BC, Green PJ. Elucidation of the small RNA component of the transcriptome. Science. 2005:309(5740):1567-9.

15. Mette MF, Aufsatz W, van der Winden J, Matzke MA, Matzke AJ. Transcriptional silencing and promoter methylation triggered by double-stranded RNA. EMBO J. 2000;19(19):5194-201. 
16. Shivaprasad PV, Dunn RM, Santos BA, Bassett A, Baulcombe DC. Extraordinary transgressive phenotypes of hybrid tomato are influenced by epigenetics and small silencing RNAs. EMBO J. 2012;31(2):257-66.

17. Chan SW, Zilberman D, Xie Z, Johansen LK, Carrington JC, Jacobsen SE. RNA silencing genes control de novo DNA methylation. Science. 2004;303(5662):1336

18. Hamilton A, Voinnet $O$, Chappell L, Baulcombe D. Two classes of short interfering RNA in RNA silencing. EMBO J. 2002;21(17):4671-9.

19. Luo Y, Guo Z, Li L. Evolutionary conservation of microRNA regulatory programs in plant flower development. Dev Biol. 2013;380(2):133-44.

20. Spanudakis $E$, Jackson $S$. The role of microRNAs in the control of flowering time. J Exp Bot. 2014;65(2):365-80.

21. Hwan LJ, Joon KJ, Ahn JH. Role of SEPALLATA3 (SEP3) as a downstream gene of miR156-SPL3-FT circuitry in ambient temperature-responsive flowering. Plant Signal Behav. 2012;7(9):1151-4.

22. Xing S, Salinas M, Hohmann S, Berndtgen R, Huijser P. miR156targeted and nontargeted SBP-box transcription factors act in concert to secure male fertility in Arabidopsis. Plant Cell. 2010;22(12):3935-50.

23. Millar AA, Gubler F. The Arabidopsis GAMYB-like genes, MYB33 and MYB65, are microRNA-regulated genes that redundantly facilitate anther development. Plant Cell. 2005;17(3):705-21.

24. Wang $Y$, Sun F, Cao H, Peng H, Ni Z, Sun Q, et al. TamiR159 directed wheat TaGAMYB cleavage and its involvement in anther development and heat response. Plos One. 2012;7(11):e48445.

25. Liang Y, Tan ZM, Zhu L, Niu QK, Zhou JJ, Li M, et al. MYB97, MYB101 and MYB120 function as male factors that control pollen tube-synergid interaction in Arabidopsis thaliana fertilization. Plos Genet. 2013;9(11):e1003933.

26. Chen X. A microRNA as a translational repressor of APETALA2 in Arabidopsis flower development. Science. 2004;303(5666):2022-5.

27. Wollmann H, Mica E, Todesco M, Long JA, Weigel D. On reconciling the interactions between APETALA2, miR172 and AGAMOUS with the ABC model of flower development. Development. 2010;137(21):3633-42.

28. Zhu QH, Helliwell CA. Regulation of flowering time and floral patterning by miR172. J Exp Bot. 2011;62(2):487-95.

29. Nag A, King S, Jack T. miR319a targeting of TCP4 is critical for petal growth and development in Arabidopsis. Proc Natl Acad Sci U S A. 2009;106(52):22534-9.

30. Pajoro A, Madrigal P, Muino JM, Matus JT, Jin J, Mecchia MA, et al. Dynamics of chromatin accessibility and gene regulation by MADS domain transcription factors in flower development. Genome Biol. 2014:15(3):R41.

31. Liang G, He H, Li Y, Wang F, Yu D. Molecular mechanism of microRNA396 mediating pistil development in arabidopsis. Plant Physiol. 2014;164(1):249-58.

32. Zhai J, Liu J, Liu B, Li P, Meyers BC, Chen X, et al. Small RNA-directed epigenetic natural variation in Arabidopsis thaliana. Plos Genet. 2008;4(4):e1000056.

33. Wei M, Wei $H$, Wu M, Song M, Zhang J, Yu J, et al. Comparative expression profiling of miRNA during anther development in genetic male sterile and wild type cotton. BMC Plant Biol. 2013;13:66.

34. Pei H, Ma N, Chen J, Zheng Y, Tian J, Li J, et al. Integrative analysis of miRNA and mRNA profiles in response to ethylene in rose petals during flower opening. PLOS One. 2013;8(5):e64290.

35. Zhang J, Wu T, Li L, Han S, Li X, Zhang S, et al. Dynamic expression of small RNA populations in larch (Larix leptolepis). Planta. 2013;237(1):89-101.

36. Wan LC, Zhang H, Lu S, Zhang L, Qiu Z, Zhao Y, et al. Transcriptomewide identification and characterization of miRNAs from Pinus densata. BMC Genomics. 2012;13:132.

37. Lu S, Sun YH, Amerson H, Chiang VL. MicroRNAs in loblolly pine (Pinus taeda L.) and their association with fusiform rust gall development. Plant J. 2007;51(6):1077-98.

38. Zhang J, Zhang S, Han S, Li X, Tong Z, Qi L. Deciphering small noncoding RNAs during the transition from dormant embryo to germinated embryo in Larches (Larix leptolepis). PLOS One. 2013;8(12):e81452

39. Zhang J, Zhang S, Han S, Wu T, Li X, Li W, et al. Genome-wide identification of microRNAs in larch and stage-specific modulation of 11 conserved microRNAs and their targets during somatic embryogenesis. Planta. 2012;236(2):647-57.
40. Quinn CR, Iriyama R, Fernando DD: Expression patterns of conserved microRNAs in the male gametophyte of loblolly pine (Pinus taeda). Plant Reprod 2014;27(2):69-78.

41. Oh TJ, Wartell RM, Cairney J, Pullman GS. Evidence for stage-specific modulation of specific microRNAs (miRNAs) and miRNA processing components in zygotic embryo and female gametophyte of loblolly pine (Pinus taeda). New Phytol. 2008;179(1):67-80.

42. Niu SH, Li ZX, Yuan HW, Chen XY, Li Y, Li W. Transcriptome characterisation of Pinus tabuliformis and evolution of genes in the Pinus phylogeny. BMC Genomics. 2013;14(1):263.

43. Nystedt B, Street NR, Wetterbom A, Zuccolo A, Lin YC, Scofield DG, et al. The Norway spruce genome sequence and conifer genome evolution. Nature. 2013:497(7451):579-84.

44. Liu Q, Feng Y, Zhu Z. Dicer-like (DCL) proteins in plants. Funct Integr Genomics. 2009;9(3):277-86.

45. Chitwood DH, Timmermans MC. Small RNAs are on the move. Nature. 2010:467(7314):415-9.

46. Dolgosheina EV, Morin RD, Aksay G, Sahinalp SC, Magrini V, Mardis ER, et al. Conifers have a unique small RNA silencing signature. RNA. 2008;14(8):1508-15.

47. Wan LC, Wang F, Guo X, Lu S, Qiu Z, Zhao Y, et al. Identification and characterization of small non-coding RNAs from Chinese fir by high throughput sequencing. BMC Plant Biol. 2012;12(1):146.

48. Vaucheret H. Plant ARGONAUTES. Trends Plant Sci. 2008;13(7):350-8.

49. Mallory A, Vaucheret $\mathrm{H}$. Form, function, and regulation of ARGONAUTE proteins. Plant Cell. 2010;22(12):3879-89.

50. Wang JW, Czech B, Weigel D. miR156-regulated SPL transcription factors define an endogenous flowering pathway in Arabidopsis thaliana. Cell. 2009;138(4):738-49.

51. Allen RS, Li J, Alonso-Peral MM, White RG, Gubler F, Millar AA. MicroR159 regulation of most conserved targets in Arabidopsis has negligible phenotypic effects. Silence. 2010;1(1):18.

52. Xie Z, Kasschau KD, Carrington JC. Negative feedback regulation of DicerLike1 in Arabidopsis by microRNA-guided mRNA degradation. Curr Biol. 2003;13(9):784-9.

53. Schommer C, Palatnik JF, Aggarwal P, Chetelat A, Cubas P, Farmer EE, et al. Control of jasmonate biosynthesis and senescence by miR319 targets. PLOS Biol. 2008;6(9):e230.

54. Rodriguez RE, Mecchia MA, Debernardi JM, Schommer C, Weigel D, Palatnik JF. Control of cell proliferation in Arabidopsis thaliana by microRNA miR396. Development. 2010;137(1):103-12.

55. Wang L, Gu X, Xu D, Wang W, Wang H, Zeng M, et al. miR396-targeted AtGRF transcription factors are required for coordination of cell division and differentiation during leaf development in Arabidopsis. J Exp Bot. 2011;62(2):761-73.

56. Mi S, Cai T, Hu Y, Chen Y, Hodges E, Ni F, et al. Sorting of small RNAs into Arabidopsis argonaute complexes is directed by the $5^{\prime}$ terminal nucleotide. Cell. 2008;133(1):116-27.

57. Bargsten JW, Folta A, Mlynarova L, Nap JP. Snf2 family gene distribution in higher plant genomes reveals DRD1 expansion and diversification in the tomato genome. PLOS One. 2013:8(11):e81147.

58. Jover-Gil S, Candela H, Robles P, Aguilera V, Barrero JM, Micol JL, et al. The microRNA pathway genes AGO1, HEN1 and HYL1 participate in leaf proximal-distal, venation and stomatal patterning in Arabidopsis. Plant Cell Physiol. 2012;53(7):1322-33.

59. Li S, Yang X, Wu F, He Y. HYL1 controls the miR156-mediated juvenile phase of vegetative growth. J Exp Bot. 2012;63(7):2787-98.

60. Addo-Quaye C, Snyder JA, Park YB, Li YF, Sunkar R, Axtell MJ. Sliced microRNA targets and precise loop-first processing of MIR319 hairpins revealed by analysis of the Physcomitrella patens degradome. RNA. 2009;15(12):2112-21.

61. Zemach A, McDaniel IE, Silva P, Zilberman D. Genome-wide evolutionary analysis of eukaryotic DNA methylation. Science. 2010;328(5980):916-9.

62. Matzke M, Kanno T, Huettel B, Daxinger L, Matzke AJ. Targets of RNAdirected DNA methylation. Curr Opin Plant Biol. 2007;10(5):512-9.

63. Vanyushin BF. DNA methylation in plants. Curr Top Microbiol Immunol. 2006;301:67-122

64. Hutvagner G, Simard MJ. Argonaute proteins: key players in RNA silencing Nat Rev Mol Cell Biol. 2008;9(1):22-32

65. Agorio A, Vera P. ARGONAUTE4 is required for resistance to Pseudomonas syringae in Arabidopsis. Plant Cell. 2007;19(11):3778-90. 
66. Zilberman D, Cao X, Jacobsen SE. ARGONAUTE4 control of locus-specific siRNA accumulation and DNA and histone methylation. Science. 2003;299(5607):716-9.

67. Tahir M, Law DA, Stasolla C. Molecular characterization of PgAGO, a novel conifer gene of the Argonaute family expressed in apical cells and required for somatic embryo development in spruce. Tree Physiol. 2006;26(10):1257-70.

68. Sohpal VK, Dey A, Singh A. MEGA biocentric software for sequence and phylogenetic analysis: a review. Int J Bioinform Res Appl. 2010;6(3):230-40.

69. Edgar RC. MUSCLE: multiple sequence alignment with high accuracy and high throughput. Nucleic Acids Res. 2004;32(5):1792-7.

70. Addo-Quaye C, Eshoo TW, Bartel DP, Axtell MJ. Endogenous siRNA and miRNA targets identified by sequencing of the Arabidopsis degradome. Curr Biol. 2008;18(10):758-62.

71. German MA, Luo S, Schroth G, Meyers BC, Green PJ. Construction of Parallel Analysis of RNA Ends (PARE) libraries for the study of cleaved miRNA targets and the RNA degradome. Nat Protoc. 2009:4(3):356-62.

72. Shuai $P$, Liang D, Zhang Z, Yin W, Xia X. Identification of drought-responsive and novel Populus trichocarpa microRNAs by high-throughput sequencing and their targets using degradome analysis. BMC Genomics. 2013;14:233.

73. Karlova R, van Haarst JC, Maliepaard C, van de Geest H, Bovy AG, Lammers M, et al. Identification of microRNA targets in tomato fruit development using high-throughput sequencing and degradome analysis. J Exp Bot. 2013:64(7):1863-78

74. Niu S, Yuan L, Zhang Y, Chen X, Li W. Isolation and expression profiles of gibberellin metabolism genes in developing male and female cones of Pinus tabuliformis. Funct Integr Genomics. 2014;14(4):697-705.

75. Mortazavi A, Williams BA, McCue K, Schaeffer L, Wold B. Mapping and quantifying mammalian transcriptomes by RNA-Seq. Nat Methods. 2008;5(7):621-8.

76. Ling LZ, Zhang SD. Exploring the evolutionary differences of SBP-box genes targeted by miR156 and miR529 in plants. Genetica. 2012;140(7-9):317-24.

77. Kim J, Jung JH, Reyes JL, Kim YS, Kim SY, Chung KS, et al. microRNAdirected cleavage of ATHB15 mRNA regulates vascular development in Arabidopsis inflorescence stems. PLANT J. 2005:42(1):84-94.

78. Sorin C, Declerck M, Christ A, Blein T, Ma L, Lelandais-Briere C, et al. A miR169 isoform regulates specific NF-YA targets and root architecture in Arabidopsis. New Phytol. 2014;202(4):1197-1211.

79. Bari A, Orazova S, Ivashchenko A. miR156- and miR171-binding sites in the protein-coding sequences of several plant genes. Biomed Res Int. 2013;2013:307145.

80. Knauer S, Holt AL, Rubio-Somoza I, Tucker EJ, Hinze A, Pisch M, et al. A protodermal miR394 signal defines a region of stem cell competence in the Arabidopsis shoot meristem. Dev Cell. 2013;24(2):125-32.

81. Zhu QH, Fan L, Liu Y, Xu H, Llewellyn D, Wilson I. miR482 regulation of NBS-LRR defense genes during fungal pathogen infection in cotton. PLOS One. 2013;8(12):e84390.

\section{Submit your next manuscript to BioMed Central and take full advantage of:}

- Convenient online submission

- Thorough peer review

- No space constraints or color figure charges

- Immediate publication on acceptance

- Inclusion in PubMed, CAS, Scopus and Google Scholar

- Research which is freely available for redistribution 